\title{
La gestion des crues du Rhône en amont du Léman : de la prévision à la décision
}

\author{
Javier GARCIA HERNANDEZ, Tristan BRAUCHLI, Jean-Louis BOILLAT, Anton J. SCHLEISS
}

\author{
Laboratoire de Constructions Hydrauliques (LCH), Ecole Polytechnique Fédérale de Lausanne (EPFL), \\ Station 18, CH-1015 Lausanne, Suisse \\ e-mail : javier.garciahernandez@epfl.ch,tristan.brauchli@a3.epfl.ch,jean-louis.boillat@epfl.ch,anton.schleiss@epfl.ch
}

\begin{abstract}
RÉSUMÉ. - Le projet MINERVE de prévision et de gestion des crues en amont du Léman est aujourd'hui opérationnel. Il vise la protection contre les inondations de la vallée du Rhône, dans les cantons du Valais et de Vaud, avec deux objectifs prioritaires. Le premier concerne la prévision hydrologique sur le bassin versant, pour une meilleure gestion des situations de crue en fonction de seuils progressifs d'avis, alerte et alarme. Le second repose sur une gestion préventive des aménagements hydroélectriques lorsque le besoin s'en fait sentir.
\end{abstract}

Mots clefs : Prévision hydrologique, crues, aide à la décision, gestion des aménagements hydroélectriques

\section{Flood management in the Upper Rhone River basin : from forecast to decision}

\begin{abstract}
The MINERVE project of flood forecast and management upstream from Lake Geneva is nowadays operational. It aims to protect against inundations in the Rhone Valley, through the Valais and Vaud Cantons. Two main objectives are expected. The first is the hydrological forecast in the catchment area for a better risk management during floods, with warning steps depending on different thresholds (notice, alert and alarm). The second is the preventive management of hydropower plants, when required, during heavy precipitations periods.
\end{abstract}

Key-words : Hydrological forecast, floods, decision making, hydropower plants management

\section{INTRODUCTION}

Sur le basin versant du Rhône en amont du Léman, les crues des dernières décennies ont rappelé l'importance de la gestion du risque d'inondation lors d'événements exceptionnels, susceptibles d'occasionner des dommages consécutifs au débordement des cours d'eau et des bassins d'accumulation. Ainsi, lors de la crue de septembre 1993, des inondations catastrophiques se sont produites dans la région du Haut-Valais, en particulier en ville de Brigue et dans la vallée de Saas, avec des dommages pour 500 millions CHF environ. En octobre 2000, la région du Bas-Valais ainsi que de nombreuses localités en amont, celle de Gondo en particulier, furent affectées, avec un coût total de plus de 500 millions CHF.

Ces événements ont aussi démontré que la capacité de rétention et de régulation des grands barrages est capable de laminer significativement les crues sur l'ensemble du réseau hydrographique, à condition de pouvoir s'appuyer sur une interprétation fiable des prévisions météorologiques. Le projet MINERVE [1] [2] [3] poursuit les objectifs de gestion préventive des crues et de déclenchement d'alertes. Il s'appuie sur un outil de simulation hydrologique basé sur les prévisions météorologiques ainsi que sur un outil d'aide à la décision. Ce dernier est capable de proposer des scénarios de turbinage et de vidange préventifs pour optimiser l'effet de laminage des retenues. Les résultats de simulation fournissent les informations utiles à la décision et à la coordination des actions à entreprendre en cas de catastrophe annoncée.

Le système MINERVE est intégré à la procédure de gestion des crues du Rhône (Figure 1). L'objectif est d'établir une planification et une coordination en tenant compte de la situation présente du bassin versant (Rhône et affluents, aménagements hydroélectriques) ainsi que des prévisions hydro-météorologiques. Sur la base de cette information, l'organe décisionnel détermine le niveau d'intervention et déclenche les mesures adéquates.

Dès qu'une situation critique est identifiée, le modèle d'aide à la décision de MINERVE propose des stratégies d'intervention pour la gestion préventive des aménagements hydroélectriques. Le but est l'empêchement ou la réduction des inondations sur le bassin versant, en conformité des objectifs pré-établis et en tenant compte des contraintes existantes. Le système expert [4] [5] poursuit deux objectifs. Le premier vise à libérer préventivement un volume de stockage dans les retenues et à interrompre les opérations de turbinage et/ou de vidange pendant la pointe de crue. Le deuxième consiste à optimiser la reconstitution des stocks à la fin de la crue, de façon à éviter les pertes d'eau.

L'utilisation des aménagements hydroélectriques pour le contrôle des crues est une opération délicate. Si les objectifs du décideur sont prioritairement de limiter les pointes de débit dans les cours d'eau, ils doivent aussi considérer l'économie des moyens. Il s'agit ainsi de limiter les pertes économiques liées aux opérations préventives lors des crues, sachant que les prévisions hydro-météorologiques sont entachées d'incertitude, en particulier pour ce qui concerne les précipitations. Les prévisions d'ensemble COSMO-LEPS permettent désormais d'évaluer partiellement cette incertitude.

La gestion active des barrages nécessite donc une estimation du coût des scénarios envisagés, raison pour laquelle un outil d'analyse hydro-économique a été développé. Il 


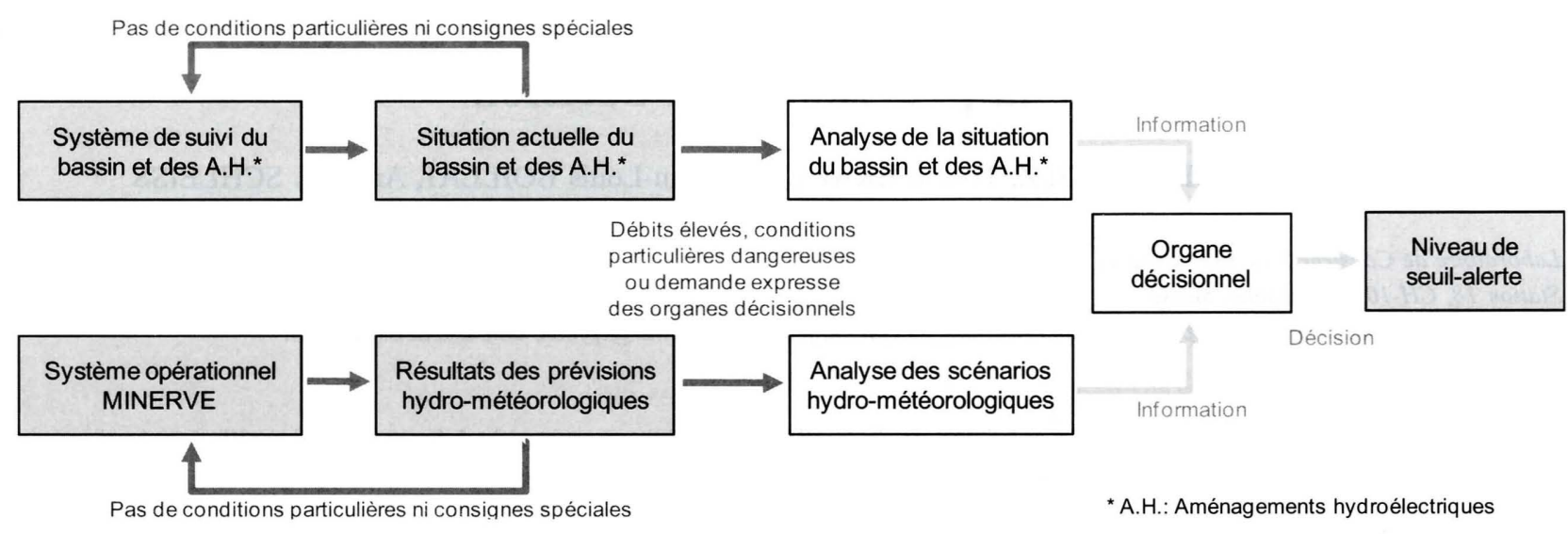

Figure 1 : Schéma opérationnel de gestion des crues du Rhône

s'intéresse d'une part aux dégâts potentiels des inondations dans la vallée du Rhône, d'autre part aux pertes économiques engendrées par les opérations de gestion préventive des aménagements hydroélectriques. Il se base sur les prévisions hydrologiques ainsi que sur les scénarios de turbinage et vidange préventifs. Il se veut un outil complémentaire dans le processus d'aide à la décision, permettant d'établir le bilan économique des pertes de turbinage et du gain sur les dommages potentiels.

\section{PRÉVISIONS HYDROMÉTÉOROLOGIQUES}

Le modèle GSM-Socont [6] est utilisé pour transformer les prévisions météorologiques en débit. Il est intégré dans l'outil de simulation hydrologique et hydraulique Routing System [7]. Ce logiciel, conçu pour simuler la formation et la propagation des écoulements dans des systèmes à surface libre, permet la prise en compte des processus hydrologiques, le routage dans les retenues, les galeries et les cours d'eau ainsi que la modélisation d'ouvrages régulés tels que vannes, évacuateurs réglables, prises d'eau, turbines ou pompes.

Le système MINERVE utilise les débits observés, les données des réservoirs et des aménagements hydroélectriques ainsi que les prévisions d'ensemble COSMO-LEPS (C-L) et les prévisions déterministes COSMO-7 (C-7) et COSMO-2 (C-2) de l'Office fédéral de météorologie et de climatologie, MétéoSuisse. Le modèle hydrologique semi-distribué contient 239 sous-bassins divisés en 1050 bandes d'altitude pour la prise en compte des processus liés à la température. Le système établit la prévision de crues en temps réel, couplant les informations météorologiques avec le modèle hydrologique. Le suivi en temps réel des écoulements est effectué sur la base des mesures enregistrées, également utilisées pour la mise à jour des variables d'état du modèle afin d'éviter la dérive de la prévision hydrologique.

Dans la perspective d'évaluer les performances du système, plusieurs resimulations d'événements passés ont été réalisées chez MétéoSuisse. Elles ont servi à une meilleure compréhension et à l'amélioration du système global ainsi qu'à l'analyse et la présentation des résultats aux utilisateurs décisionnels.

Les résultats de trois événements étudiés (septembre 1993, octobre 2000 et mai 2008) permettent une comparaison entre C-L et C-7. Ils ont donné un biais de débit relatif [8] de 0.49 en moyenne pour des périodes journalières dans le cas de C-7 et de 0.19 pour la médiane de C-L. L'erreur moyenne sur le temps de prévision de la pointe est de $8 \mathrm{~h}$ pour C-7 et de $6 \mathrm{~h}$ pour la médiane de C-L, et l'erreur normalisée du débit de pointe [9] est de 0.19 pour C-7 et 0.24 pour la médiane de C-L. D'autres indicateurs probabilistes comme le Brier Score [10] ou le ROC [11] donnent de meilleures performances à la prévision d'ensemble C-L en comparaison de C-7, plus variable en général.

Un suivi de la crue d'octobre 2000, avec les prévisions hydro-météorologiques provenant de resimulations météorologiques, est présenté à titre d'exemple sur la Figure 2 . La crue est assez bien prévue par COSMO-LEPS trois jours avant le débit de pointe, avec la meilleure performance correspondant au quartile supérieur $\mathrm{q}=75 \%$. COSMO-7 se révèle plus instable, en prévoyant le débit de pointe en avance pour plusieurs prévisions. Il conduit également à une sous- ou surestimation du débit de pointe selon les cas.

\section{BULLETIN D'AVERTISSEMENT}

Avant l'activation du modèle d'aide à la décision, le bulletin d'avertissement MINERVE (Figure 3) montre de manière synthétique l'évolution de la situation hydrologique aux différentes points de contrôle du bassin versant et donne des messages d'avertissement (avis, alerte ou alarme) en fonction de différents seuils de dépassement de débit (Tableau 1) et de probabilité des prévisions d'ensemble. Les points de contrôle choisis sont répartis sur l'ensemble du bassin versant dans le cours principal du Rhône (Reckingen, Brig, Sion, Branson, Lavey et Porte du Scex) et sur ses deux affluents principaux (Vispa et Dranse).

Pour le déclenchement d'un avertissement de COSMOLEPS, l'hydrogramme correspondant à la médiane $\mathrm{q}=50 \%$ a été sélectionné. Ce seuil de COSMO-LEPS pourrait toutefois être différencié pour chaque niveau d'avertissement, selon les résultats de performance des prévisions.

\section{GESTION DES CRUES}

\section{IV.1. Procédure de gestion des crues dans les cantons du Valais et de Vaud}

Dès qu'une situation exceptionnelle est détectée, la procédure de gestion des crues du Rhône est déclenchée dans les cantons du Valais (VS) et de Vaud (VD) [12]. Les débits aux points de contrôle, la situation générale de remplissage des barrages et du bassin versant ainsi que les prévisions hydrométéorologiques fournissent aux décideurs l'information de 

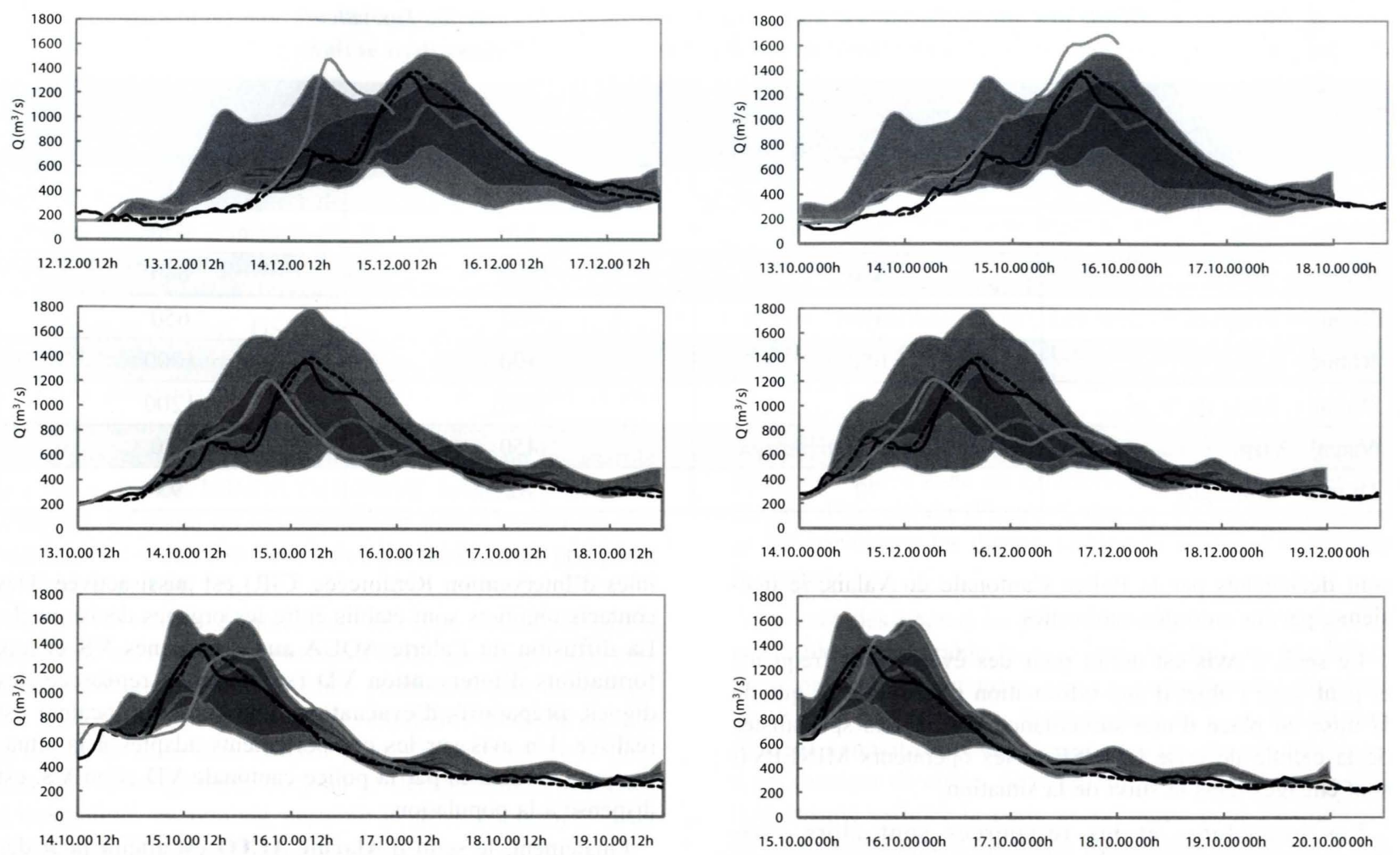

Figure 2 : Prévisions hydrologiques à la Porte du Scex pour les prévisions COSMO-LEPS (C-L) des 12, 13 et 14.10 .2000 à $12 \mathrm{~h} 00$ et pour les prévisions COSMO-7 (C-7) des 12, 13 et 14.10 .2000 à $12 \mathrm{~h} 00$ et 13, 14 et 15.10 .2000 à $00 \mathrm{hO0}$. Simulation avec les données météorologiques observées $(R S)$ et comparaison avec le débit observé $\left(Q_{\text {obs }}\right)$

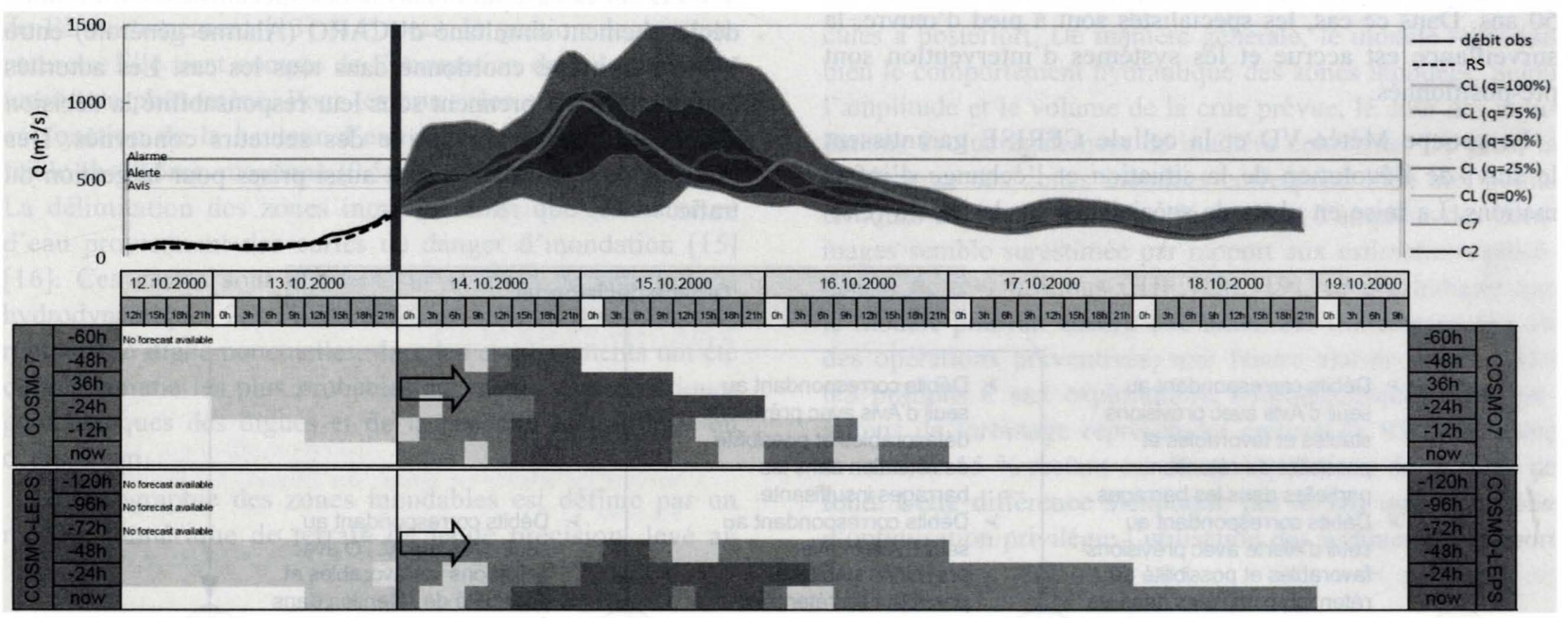

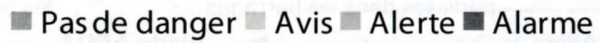

Figure 3 : Prévisions hydrologiques et bulletin d'avertissement MINERVE à Sion, selon les prévisions météorologiques COSMOLEPS du 13.10.2000 à $12 \mathrm{hO0}$ et COSMO-7 du 14.10.2000 à 00h00.

base pour définir la marche à suivre (Figure 4). La prise de décisions et les démarches associées dépendent des éléments suivants :

- débits observés aux points de contrôle (supérieurs ou inférieurs aux débits seuil),

- prévisions de précipitations (à la baisse, stable ou à la hausse),

- prévisions hydrologiques MINERVE (favorables, stables ou défavorables) et
- possibilités de rétention dans les barrages (complètes, partielles ou insuffisantes)

En fonction de la situation prévue, un des seuils d'avertissement, définis par le Service de Routes et de Cours d'Eau du Canton du Valais (SRCE) en coordination avec le Service des Eaux, Sols et Assainissement du Canton de Vaud (SESA) et le Service de la sécurité civile et militaire du Canton de Vaud (SSCM), peut être atteint aux points de contrôle. Les deux premiers seuils d'Avis et d'Alerte AQUA 
Tableau 1: Débits des différents seuils d'avertissement aux points de contrôle. Les valeurs sont indicatives, en attente de validation par l'Etat du Valais.

\begin{tabular}{|c|c|c|c|c|c|}
\hline \multirow{2}{*}{ Point de contrale } & \multicolumn{2}{|c|}{ Sectil $1\left(\mathrm{~m}^{3} / \mathrm{s}\right)$} & Sevil $2\left(\mathrm{ni}^{3} / 5\right)$ & \multicolumn{2}{|c|}{ euti $3\left(\mathrm{~m}^{3} / \mathrm{s}\right)$} \\
\hline & Avis & & Alerte & Alarme & \\
\hline Rhône - Reckingen & 75 & $x^{2}$ & 95 & 115 & $x^{2}$ \\
\hline Rhône - Brig & 245 & & 340 & 430 & \\
\hline Rhône - Sion & 450 & & 530 & 640 & \\
\hline Rhône - Branson & 475 & & 550 & 650 & \\
\hline Rhône - Lavey & 650 & 2 & 800 & 1000 & $x$ \\
\hline Rhône - Porte du Scex & 700 & & 1000 & 1200 & \\
\hline Vispa - Visp & 370 & 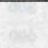 & 450 & 550 & Co \\
\hline Dranse - Martigny & 70 & & 82 & 95 & \\
\hline
\end{tabular}

sont déclenchés par la Police Cantonale du Valais, le troisième, par les autorités cantonales.

Le seuil d'Avis est défini pour des événements fréquents et peut faire l'objet d'une information générale ainsi que de la mise en place d'une surveillance légère. Les spécialistes de la cellule de crise CERISE et les opérateurs MINERVE sont engagés dans le suivi de la situation.

Les procédures et les ressources sont alors vérifiées et l'information est transmise aux autres partenaires (Groupe météo-VD). Le SRCE-VS informe aussi le SESAVD et le Domaine de l'eau (département du territoire) de Genève (DomEAU-GE).

Le seuil d'Alerte AQUA est déclenché pour un événement peu fréquent, de période de retour comprise entre 20 et 50 ans. Dans ce cas, les spécialistes sont à pied d'œuvre, la surveillance est accrue et les systèmes d'intervention sont pré-positionnés.

Le groupe Météo-VD et la cellule CERISE garantissent le suivi de l'évolution de la situation et l'échange d'informations. La mise en place de spécialistes sur le terrain (Cel- lules d'Intervention Renforcées, CIR) est aussi activée. Des contacts réguliers sont établis entre les organes décisionnels La diffusion de l'alerte AQUA aux communes VS et aux formations d'intervention VD (surveillance renforcée des digues, préparatifs d'évacuation, interventions locales) est réalisée. Un avis sur les comportements adaptés à la situation, et coordonnés par la police cantonale VD et/ou VS, est dispensé à la population.

Finalement, le seuil d'Alarme ALTO est atteint pour des événements rares (période de retour de 100 ans). Il permet d'activer les plans d'alarme et d'évacuation de populations, adaptés au risque et à la situation.

Arrivé à ce stade, une évacuation préventive des zones concernées peut être décidée par les autorités cantonales VS-VD sur la base de l'analyse des spécialistes. Un éventuel déclenchement simultané d'ICARO (Alarme générale) entre VD-VS doit être coordonné dans tous les cas. Les autorités communales VS prennent sous leur responsabilité la décision d'une évacuation préventive des secteurs concernés. Des mesures de coordination sont aussi prises pour la gestion du trafic.

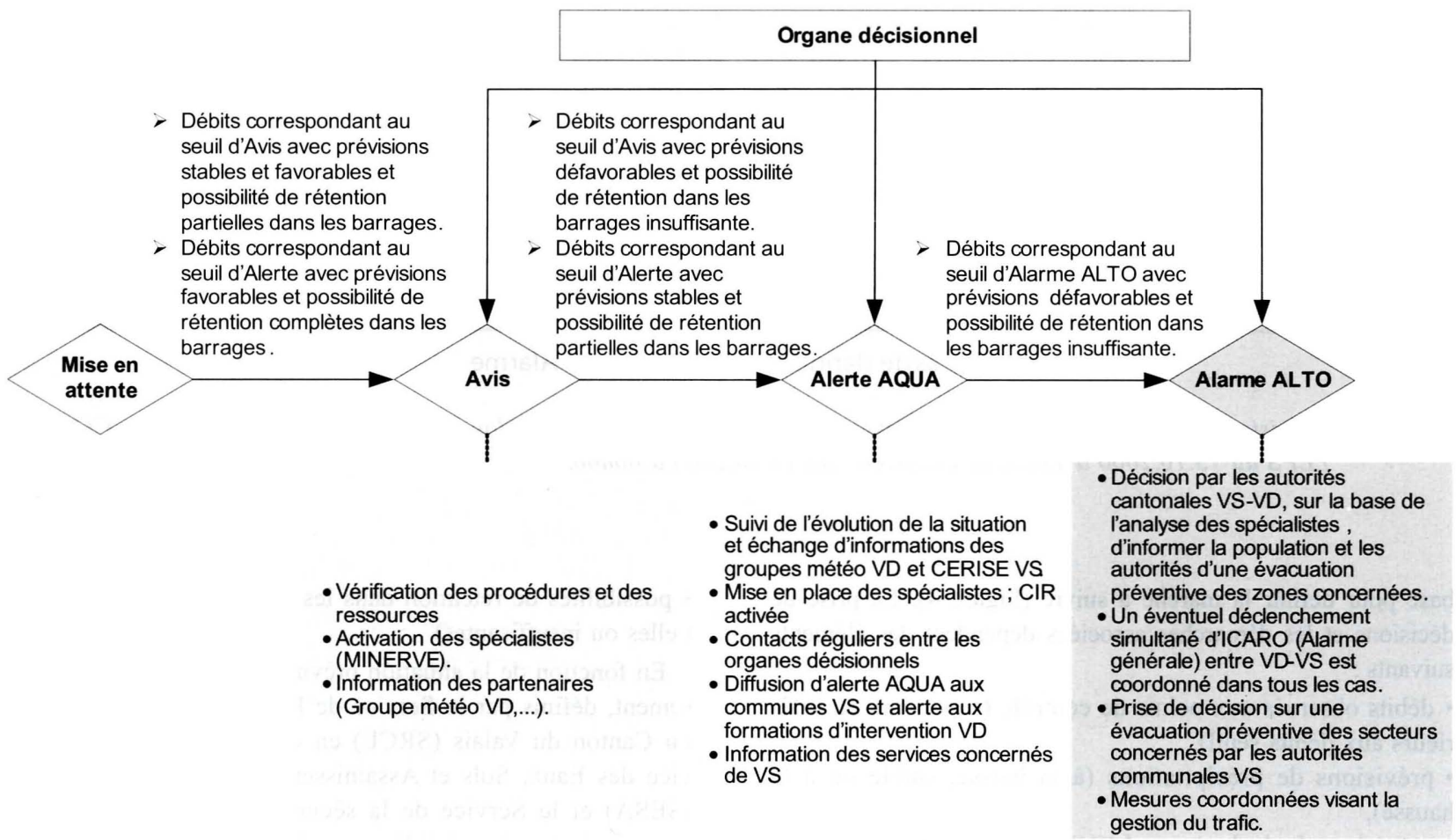

Figure 4 : Procédure d'alarme pour la gestion des crues du Rhône dans les cantons du Valais (VS) et de Vaud (VD) 


\section{IV.2. Gestion préventive des aménagements hydroélectriques et analyse hydro-économique pour l'aide à la décision}

L'analyse de risque permet d'évaluer la rationalité d'une décision, ce qui est particulièrement important pour les prévisions de crues découlant de prévisions hydro-météorologiques chargées d'incertitude. L'évaluation du risque est ainsi nécessaire en prélude à une décision. Cette dernière peut s'appuyer sur un système d'aide à la décision (Decision Support System, DSS), outil conçu pour l'évaluation de différents scénarios dépendant de valeurs estimées. Le DSS est indispensable à l'optimisation de systèmes complexes de réservoirs à objectifs multiples, dédiés à la production d'électricité, l'approvisionnement en eau ou le contrôle des crues. L'outil MINDS (MINERVE Interactive Décision Support), en cours de développement pour l'évaluation des scénarios de crue, vise l'évolution probabiliste du précédent système expert [4] [5].

Les prévisions d'ensemble de type COSMO-LEPS apportent un éclairage nouveau à cette question, avec certains avantages potentiels. Elles permettent une évaluation de l'incertitude associée aux événements considérés, qui peut par la suite être utilisée pour l'évaluation et la gestion des aménagements ou pour le déclenchement d'alertes d'inondation, en rendant possible la prise en compte d'un risque probabiliste.

Quand le seuil d'Avis est dépassé, les outils d'aide à la décision et d'analyse hydro-économique sont activés. Grâce à ces outils, des estimations peuvent être fournies sur le coût potentiel des inondations et le coût des opérations préventives en cas de fausse alarme. La première de ces estimations se base sur la méthode définie par l'Office Fédéral de l'Environnement [13] [14] pour la gestion des risques naturels. Elle tient compte de l'occupation du sol et de l'intensité du phénomène. Pour les crues, les coûts sont définis en fonction de la hauteur d'eau dans les zones inondées: faible $(\mathrm{h}<0.5 \mathrm{~m})$, moyenne $(0.5 \mathrm{~m}<\mathrm{h}<2 \mathrm{~m})$ et forte $(\mathrm{h}>2 \mathrm{~m})$. La délimitation des zones inondées ainsi que les hauteurs d'eau proviennent des cartes de danger d'inondation [15] [16]. Ces cartes sont établies sur la base de simulations hydrodynamiques $2 \mathrm{D}$. Les inondations virtuelles résultent de ruptures de digue ponctuelles, dont les emplacements ont été définis comme les plus probables au vu des caractéristiques géotechniques des digues et de la capacité hydraulique du cours d'eau.

La topographie des zones inondables est définie par un modèle numérique de terrain de haute précision, levé au laser par Swisstopo [17]. Cette technique est celle qui permet, à l'heure actuelle, d'avoir la meilleure précision. La taille de la maille est de $2 \mathrm{~m}$ et la précision altimétrique de $0.5 \mathrm{~m}$. Finalement, l'occupation du sol provient du plan d'affectation des zones (PAZ). Ce plan est l'outil de gestion du développement territorial établi par les autorités locales.

Jusqu'ici, 25 zones inondables (Figure 5, gauche) ont été définies entre la ville de Sierre et le Léman, couvrant une surface de $87 \mathrm{~km}^{2}$ et dont le volume de rétention équivaut à environ 155 millions de $\mathrm{m}^{3}$. Les zones inondables à l'amont de Sierre, actuellement à l'étude, compléteront le système. L'ouverture d'une brèche et le débit déversé dans la plaine sont calculés sur la base de la prévision hydrologique et dépendent de la capacité hydraulique du fleuve. Schématiquement, chaque zone est caractérisée par une brèche et une surface d'inondation (Figure 5, droite). Le retour au Rhône se fait par-dessus les digues, lorsque le niveau d'inondation le permet.

Le coût des opérations préventives se base sur le scénario le plus défavorable, considérant qu'elles ont été effectuées préventivement mais que les précipitations attendues ne se sont pas produites. Pour évaluer ce coût, il faut tout d'abord connaître les caractéristiques de chaque aménagement (hauteur de chute, débit équipé et débit de vidange). Il faut ensuite estimer la perte économique pour l'exploitant, en fonction du type d'opération demandé. Celui-ci est fixé à $0.06 €$ par $\mathrm{kWh}$ pour le turbinage préventif et $0.09 €$ par $\mathrm{kWh}$ lors de l'utilisation de la vidange de fond.

Afin d'illustrer le potentiel de l'outil d'aide à la décision, une analyse hydro-économique de la crue d'octobre 2000 a été réalisée. Elle se base sur les prévisions hydrologiques et les scénarios de gestion préventive des aménagements, calculés a posteriori. De manière générale, le modèle reproduit bien le comportement hydraulique des zones inondées. Selon l'amplitude et le volume de la crue prévue, le coût des inondations évolue également de manière cohérente. De plus, la localisation des zones inondées correspond bien aux points critiques du bassin versant. Néanmoins, l'ampleur des dommages semble surestimée par rapport aux estimations précédentes de 500 millions CHF [18] [19], ce qui indique que le modèle pourrait encore être amélioré. Concernant le coût des opérations préventives, une fausse alarme aurait coûté 6.3 millions $€$ aux exploitations hydroélectriques. Les opérations de turbinage représentent environ le $85 \%$ de cette somme, le $15 \%$ restant est dû aux opérations de vidange de fond. Cette différence s'explique par le fait que le modèle d'optimisation privilégie l'utilisation des turbines par rapport
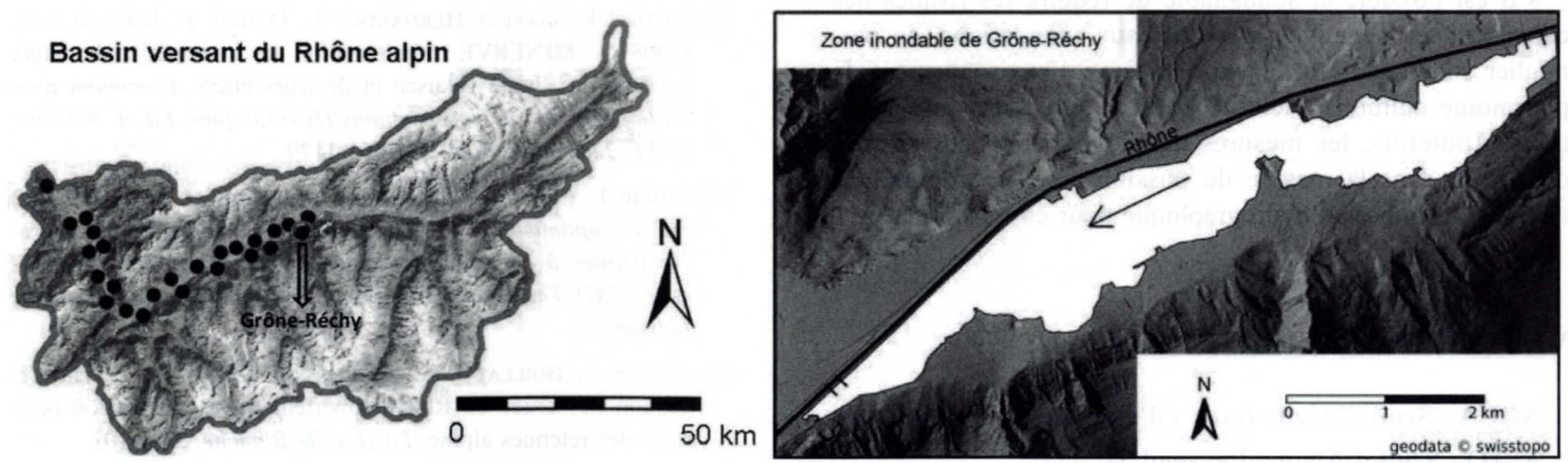

Figure 5 : Localisation des principales zones inondables à l'aval de Sierre (points noirs) sur le bassin versant du Rhône alpin (à gauche). Exemple de la zone inondable de Grône-Réchy (à droite). Les doubles traits indiquent l'emplacement de la brèche et du retour au Rhône. La flèche indique le sens de l'écoulement 
aux vidanges de fond. Comparés aux coûts induits par les inondations, les coûts des opérations préventives sont nettement plus faibles (autour de $1 \%$ ). En cas de fausse alarme, ils sont pourtant loin d'être négligeables, ce qui confirme l'importance de la fiabilité du système d'aide à la décision.

En cas de prévision hydrologique pessimiste, l'outil d'analyse hydro-économique apporte une information supplémentaire. Jusqu'à présent, le décideur ne disposait d'aucune évaluation de l'impact économique de ses choix. Ce nouveau modèle fournit en temps réel une estimation quantitative des coûts induits et permet de combler en partie le déficit d'information. Les scénarios développés constituent une base solide pour établir un critère de décision lors d'interventions préventives. En combinant le coût des inondations potentielles et la perte induite par la gestion préventive des aménagements, le décideur connait le gain qu'il peut espérer et agir en conséquence. Dans certains cas, la gestion préventive est la meilleure solution alors que dans d'autres, il est préférable de ne rien faire. Les résultats du modèle d'analyse hydro-économique constituent une base importante pour la prise de décision.

\section{CONCLUSION ET DISCUSSION}

La procédure de gestion des crues du Rhône, approuvée officiellement en 2009, est actuellement appliquée. Le système MINERVE, élément important de la procédure de décision, est opérationnel depuis 2006. Il s'appuie sur les prévisions météorologiques COSMO-7 et depuis 2008 les développements en cours peuvent également bénéficier des prévisions COSMO-LEPS et COSMO-2 établies par MétéoSuisse. Il contribue à la prise de décisions prioritaires pour la gestion préventive des aménagements hydroélectriques dans un but sécuritaire tenant compte des coûts et des risques potentiels d'inondation. Il permet d'établir un tableau synoptique de la situation hydro-météorologique sur l'ensemble du bassin versant.

La création d'un site web, rassemblant l'information sur la situation présente du bassin versant (débits des rivières, niveaux des retenues d'accumulation, remarques caractéristiques,...) et l'information issue du système MINERVE (prévisions hydro-météorologiques, bulletin d'avertissement, gestion des barrages,...) est en cours de développement pour faciliter la communication.

Une bonne procédure implique une coordination à l'échelle du bassin hydrologique, comme indiqué dans la directive européenne [20], qui considère que :

« Il est possible et souhaitable de réduire les risques des conséquences négatives associées aux inondations, en particulier sur la santé et la vie humaines, l'environnement, le patrimoine culturel, l'activité économique et les infrastructures. Toutefois, les mesures de réduction de ces risques devraient, dans la mesure du possible, être coordonnées à l'échelle d'un bassin hydrographique pour être efficaces. »

\section{NOMENCLATURE}

AQUA : Seuil d'Alerte (ou seuil 2)

ALTO : Seuil d'Alarme (ou seuil 3)

CERISE: Cellule scientifique de crise du Canton du Valais

CIR : Cellules d'Intervention Renforcées
COSMO-LEPS (C-L) : Consortium for Small scale Modeling - limited-area Ensemble Prediction System

COSMO-2 (C-2) : Consortium for Small-scale Modeling (2.2 $\mathrm{km}$ de résolution)

COSMO-7 (C-7) : Consortium for Small-scale Modeling (6.6 $\mathrm{km}$ de résolution)

DomEAU-GE : Domaine de l'eau (département du territoire) du canton de Genève

ICARO : Information Catastrophe Alarme Radio Organisation

MINERVE : Modélisation des Intempéries de Nature Extrême dans le Rhône valaisan et de leurs effets

MINDS : MINERVE Interactive Decision Support

SESA : Service des Eaux, Sols et Assainissement du Canton de Vaud

SRCE : Service des Routes et des Cours d'Eau du Canton du Valais

SSCM : Service de la sécurité civile et militaire du Canton de Vaud

VD : Canton de Vaud

VS : Canton du Valais

\section{REMERCIEMENTS}

Le projet MINERVE est développé en partenariat par l'Office Fédéral de l'Environnement, les Services des Routes et Cours d'Eau, de l'Energie et des Forces Hydrauliques du Canton du Valais et par le Service des Eaux, Sols et Assainissement du Canton de Vaud. L'Office fédéral de météorologie et de climatologie (MétéoSuisse) fournit les prévisions météorologiques et les sociétés hydroélectriques communiquent les informations relatives à leurs aménagements. Les développements scientifiques sont confiés à deux entités de l'EPFL, le Laboratoire d'Ecohydrologie et le Laboratoire de Constructions Hydrauliques, ainsi qu'à l'Institut de Géomatique et d'Analyse du Risque de l'UNIL.

\section{RÉFÉRENCES}

[1] Garcia Hernandez J., Boillat J.-L., Jordan F., Hingray B. (2009) - La prévision hydrométéorologique sur le bassin versant du Rhône en amont du Léman. La Houille Blanche. 5 61-70

[2] Boillat J.-L. (2009) - Prévision hydrologique et aide à la décision Swiss Engineering. 7/8 10

[3] Jordan F., Garcia Hernandez J., Dubois J., Boillat J.-L. (2008) - MINERVE : Modélisation des intempéries de nature extrême du Rhône valaisan et de leurs effets. Communication du Laboratoire de Constructions Hydrauliques, Ed. A. Schleiss, EPFL, Lausanne, ISSN 38 1661-1179

[4] Jordan F. (2007) - Modèle de prévision et de gestion des crues - optimisation des opérations des aménagements hydroélectriques à accumulation pour la réduction des débits de crue. PhD Thesis $N^{\circ} 3711$, Ecole Polytechnique Fédérale de Lausanne

[5] Jordan F., Boillat J.-L., Schleiss A. (2010) — Prévision et gestion des crues du Rhône supérieur par l'exploitation optimale des retenues alpine. La Houille Blanche. 5 91-102

[6] Schäfli B., Hingray B., Niggli M., Musy A. (2005) - A conceptual glacio-hydrological model for high mountainous catchments. Hydrology and Earth System Sciences Discussions. 2 $73-117$ 
[7] Garcia Hernández J., Jordan F., Dubois J., Bolllat J.-L., Schleiss A. (2007) - Routing System II : Modélisation d'écoulements dans des systèmes hydrauliques. Communication du Laboratoire de Constructions Hydrauliques, Ed. A. Schleiss, EPFL, Lausanne $\mathbf{3 2}$

[8] Ajami N.K., Gupta H., Wagener T., Sorooshian S. (2004) Calibration of a semi-distributed hydrologic model for streamflow estimation along a river system. Journal of Hydrology. 298 112-135

[9] Gabellani S., Boni G., Ferraris L., Von Hardenberg J., Provenzale A. (2007) - Propagation of uncertainty from rainfall to runoff : A case study with a stochastic rainfall generator. Advances in Water Resources. 30 2061-2071

[10] Buizza R., Houtekamer P.L., Toth Z., Pellerin G., Wei M., ZHU Y. (2005) - A comparison of the ECMWF, MSC and NCEP global ensemble prediction systems. Monthly Weather Review. 133 1076-1097

[11] He, Y. ; Wetterhall, F. ; Cloke, H. L. ; Pappenberger, F. ; Wilson, M. ; Freer, J. ; Mcgregor, G. (2009) - Tracking the uncertainty in flood alerts driven by grand ensemble weather predictions. Meteorological Applications. 16(1) 91-101

[12] Service De La Securite Civil Et Militaire (2009) — Procédure lors de crues du Rhône pour les cantons du Valais et de Vaud. Rapport final accepté, Section organisation, planification et prévention en cas de catastrophes, Département des finances, des institutions et de la sécurité, Grône, Canton du Valais

[13] Buwal (1999) - Risikoanalyse bei gravitativen Naturgefahren: Methode. Umwelt-Materialien Nr 107/I. Bundesamt für Umwelt, Wald und Landschaft, Bern
[14] Buwal (1999) — Risikoanalyse bei gravitativen Naturgefahren: Fallbeispiele und Daten. Umwelt-Materialien Nr 107/ II. Bundesamt für Umwelt, Wald und Landschaft, Bern

[15] Stucky Ingénieurs-Conseils Sa (2005) - Etablissement de la carte des dangers du Rhône sur sa rive droite. Etat de Vaud, Service des eaux, sols et assainissements (SESA), Lausanne

[16] Ingenieurbüro Niederer + Pozzi Ag (2009) — Etablissement de la carte des dangers du Rhône. Etat du Valais

[17] Swisstopo (2005) - DTM-AV. MNT-MO Collection Géodonnées Office fédéral de topographie, Ittigen 21

[18] Srce (1999) - Troisième correction du Rhône. Projet général, données de base. Hydrologie, hydraulique fluviale, objectifs de protection, carte des dangers, dégâts potentiels. Canton du Valais, Service des routes et des cours d'eau, Sion

[19] Sesa, Srce (2006) — Troisième correction du Rhône. Rapport "Danger ", partie 1: Hydrologie, hydraulique, morphologie alluviale, objectifs de protection, dégâts potentiels et examen des dangers. Canton de Vaud, Service des eaux, sols et assainissement, Lausanne, Canton du Valais, Service des routes et des cours d'eau, Sion

[20] The European Parliament And The Council Of The European Union (2007) - DIRECTIVE 2007/60/EC of the European Parliament and the Council of the European Union of 23 October 2007 on the assessment and management of flood risks. Official Journal of the European Union. L 288 27-34 